\title{
Dark adaptation and the Pulfrich effect ${ }^{\prime}$
}

\author{
L. G. STANDING, ${ }^{2}$ P. C. DODWELL AND D. LANG \\ QUEEN'S UNIVERSITY, Kingston, Ontario
}

The Pulfrich effect (the illusory displacement of a moving stimulus when it is viewed with an optical filter over one eye) was measured repeatedly during a 20 min period following the initial filtering of the eye. For each of 10 filter densities, the Pulfrich effect always occurred immediately upon filtering the eye, but subsequently increased greatly during the adaptation period. The results indicate that the perceptual latency of the filtered eye is increased by two factors: the reduction in stimulus luminance due to the filter and progressive dark adaptation. Dark adaptation is apparently the more important of these two factors.

The phenomenal path of a moving object, viewed with a colored or neutral density filter over one eye, is displaced from its true path (Pulfrich, 1922). The size and direction of the displacements are usually accepted as evidence that perceptual latency in the filtered eye increases monotonically with the optical density of the filter employed (Lit, 1949; Ogle, 1962). This interpretation is consistent with the known inverse relation between the intensity of a visual stimulus and its perceptual latency (Pieron, 1952), and the slower speed of response of the dark-adapted eye (Granit, 1947). Electrophysiological work on visual evoked potentials supports both points (Vaughan \& Costa, 1964). Normally, when the Pulfrich effect is measured repeatedly the two factors are confounded, so that either or both may be responsible for the phenomenon. It is sometimes assumed (e.g., Gregory, 1966) that the effect is simply due to differences in adaptation level in the two eyes, but Harker, Dodwell, and Behar (1967) have shown that it can be obtained when the eyes are equally adapted. Differential adaptation of the eyes can cause displacement, however, since it has been found that, when one eye is dark adapted and $S$ then views the moving object (without filters), the Pulfrich effect occurs in a direction corresponding to greater latency in the dark-adapted eye (Engelking \& Poos, 1924; Rock \& Fox, 1949). In this case the effect attentuates quite rapidly, as the darkadapted eye approaches the level of light adaptation of the other eye. However, progressive changes in the Pulfrich effect during the course of dark adaptation have not been studied previously.

The present study is intended to determine whether progressive changes in adaptation play an appreciable role in the Pulfrich effect. This is achieved by measuring, for each of a series of filters, the size of the effect at various times from the moment when one eye is initially filtered to the time when a steady state of adaptation has been reached.

\section{Subjects}

Two of the authors (LGS and DL) served as Ss. Both possessed normal uncorrected vision.

\section{Apparatus}

The $S$ viewed a moving pointer $P$, which was mounted on a turntable and crossed his line of sight from right to left every $1.8 \mathrm{sec}$, at a distance of $140 \mathrm{~cm}$. P moved at $31.4 \mathrm{~cm} / \mathrm{sec}$. Between $S$ and $P$ a half-silvered mirror was mounted at $45 \mathrm{deg}$, so that when his head was placed in an adjustable headrest $P$ was visible through the mirror. A movable pointer $M$ was mounted so that its reflected image in the mirror lay just below the level of $\mathbf{P}$; by moving $\mathbf{M}$ along a scale perpendicular to his line of sight, $S$ could make its reflected image appear equidistant with $P$.

The $S$ viewed the stimuli through $4 \mathrm{~mm}$ artificial pupils, to limit any effects due to change in pupil size and reduce the entry of extraneous stray light to the eyes. The pointers $P$ and $M$ were narrow upright cylinders, $2 \mathrm{~cm}$ high, $3 \mathrm{~mm}$ wide, painted white and illuminated to provide luminances of $120 \mathrm{~mL}$ and $46 \mathrm{~mL}$, respectively, as measured at the eye. They were viewed against a background luminance of $6 \mathrm{~mL}$ in an otherwise dark room.
Procedure

Each $\mathrm{S}$ was given several hours of practice in viewing the Pulfrich effect before he was tested. At the start of each experimenta. session $S$ viewed the stimuli through the artificial pupils for $10 \mathrm{~min}$ to achieve a steady pre-experimental level of light adaptation. Then, at time $\mathrm{O}$, a neutral density filter was dropped into place in front of S's left eye and he instantly adjusted the pointer $M$ so that it appeared equidistant with the nearest point of the phenomenal path of the moving pointer $P$. If the initial setting could not be made within 2 or $3 \mathrm{sec}$ to $S$ 's satisfaction, the filter was removed and the procedure repeated. The $S$ then viewed the moving pointer $P$ for $20 \mathrm{~min}$, keeping the adjustable pointer $M$ adjusted to the same apparent distance. Fixation was maintained on the pointer $P$. The position of $M$ was recorded for the initial setting and at $1 \mathrm{~min}$ intervals thereafter.

For each of the two Ss used, 10 values of neutral density filter were employed, from 0.3 to $3.0 \mathrm{log}$ units in steps of 0.3 . Each value was used for 10 experimental sessions, the successive treatments for each $S$ being arranged in a 10 by 10 Latin square. About $60 \mathrm{~h}$ of testing were required for each $\mathrm{S}$.

Control readings were obtained under two conditions. In the first (control condition 1), settings of $M$ for apparent equidistance with $P$ were made under conditions identical to an experimental session, except that no filters were employed, to test for stability of depth judgments over time. In control condition $2, S$ was given a 20-min preadaptation period using one of the filters (during which he viewed the stimuli, but with $P$ stationary) followed immediately by a 20 -min experimental session using that filter, during which readings were taken of the magnitude of the Pulfrich effect. This procedure was repeated for each filter value, to check that the magnitude of the Pulfrich effect had reached a true asymptote during the preadaptation period, and that S's settings of $M$ thereafter were stable.

\section{RESULTS}

The Pulfrich effect here is measured as the difference in position of the pointer $M$ under experimental conditions from its settings made under control condition 1. In Fig. 1 the settings of

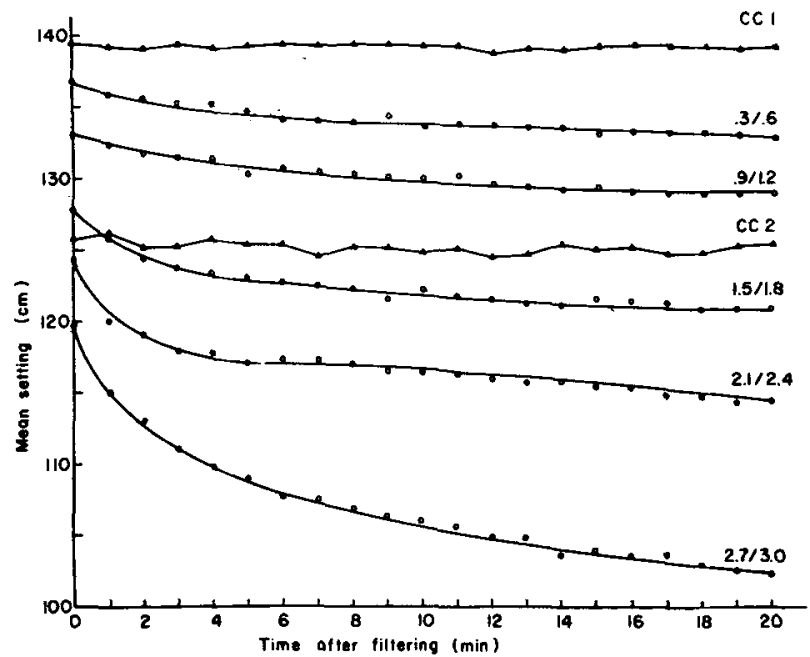

Fig. 1. Mean settings of movable pointer $M$ for apparent equidistance with moving pointer $P$, as a function of fitter density and time after filtering. Filter densities (adjacent values pooled) are given on each adaptation curve. Also shown: settings made with no filter and after preadaptation (control conditions 1 and 2). Data are pooled for both Ss, and (for control condition 2) for all filter densities. 
$M$ under experimental conditions are shown as a function of time following the initial filtering of the eye, for each filter density. These settings are given as the distance between $S$ and the pointer $\mathrm{M}$; each point in these functions is the mean of 40 observations, 20 from each S. While the Pulfrich effect was larger for Subject $\mathrm{DL}$, the overall form of the adaptation curves was very similar for both $S s$, so that their data may meaningfully be pooled.

The mean settings of $M$ under control conditions 1 and 2 are also shown in Fig. 1, pooled for both Ss. Each point is again the mean of 20 observations, readings being pooled for all filter values in the latter case.

The data for each $S$ were also analyzed separately by the analysis of variance. For both Ss, settings of $M$ were significantly affected by filter density $(\mathrm{p}<.01)$ and practice $(\mathrm{p}<.01)$. The effects of practice were not large and consisted of irregular fluctuations in the magnitude of the effect. The data are summarized in Table 1.

The mean size of the Pulfrich effect at time $O$ is plotted against its magnitude after 20 min in Fig. 2, for each filter value and each $S$. These values represent the first and last points of the functions given in Fig. 1, and indicate, respectively, the size of the effect before and after dark adaptation has occurred. Also shown on Fig. 2 are the inferred latency differences which correspond to each level of the effect, calculated from the formula of Lit (1949).

\section{DISCUSSION}

The data show that adaptational changes in the Pulfrich effect are pronounced for all filter values and for both Ss. Since adaptional changes did not occur appreciably under control conditions, and a virtually asymptotic level of the Pulfrich effect was found immediately after preadapting the eye to darkness, the systematic increases in the effect observed during each experimental session must be attributed to dark adaptation rather than to some form of practice effect.

Our results are consistent with the idea that the primary cause of the Pulfrich effect is a change in perceptual latency in one eye (Lit, 1949), and that latency increases progressively during dark adaptation. They are also consistent with the findings of Engelking

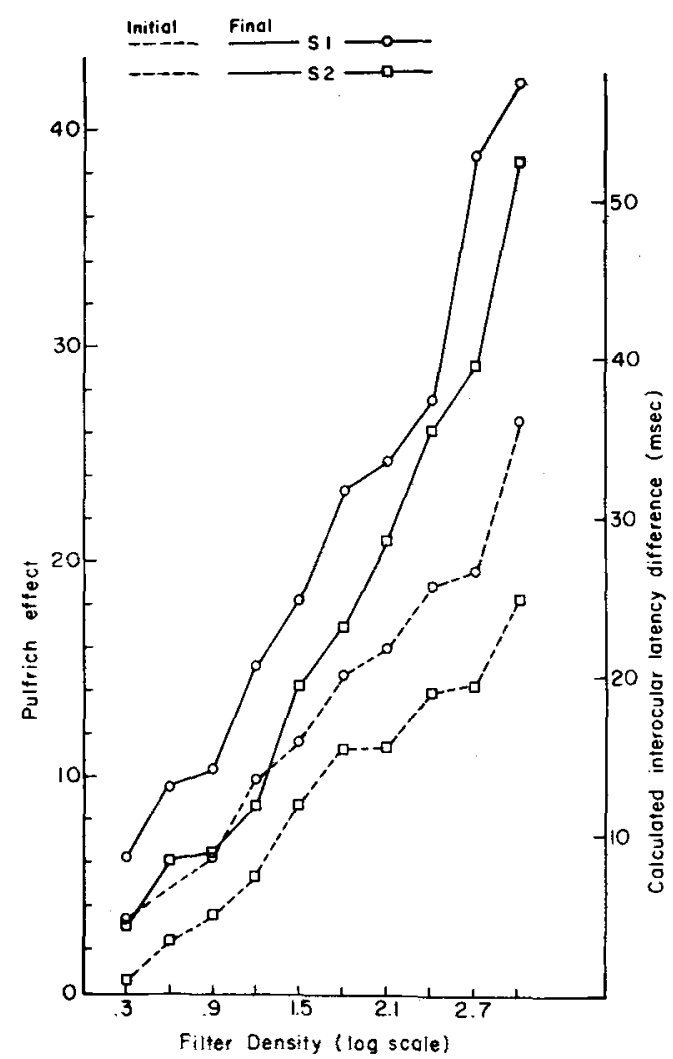

Fig. 2. Magnitude of the Pulfrich effect in centimeters before and after 20 min of dark adaptation "initial" and "final" settings) as a function of filter density, together with calculated latency differences.

Table 1

Distance $(\mathrm{cm})$ at which movable pointer $M$ was set for apparent equidistance with moving pointer $P$ under experimental conditions (dark adaptation occurring), control condition 1 (no filter employed) and control condition 2 (subject preadapted to filter).

The true distance of $P$ from $S$ was $140 \mathrm{~cm}$. Values are pooled for all

filter values under control condition 2. Upper and lower values within each pair are observations on LGS and DL, respectively.

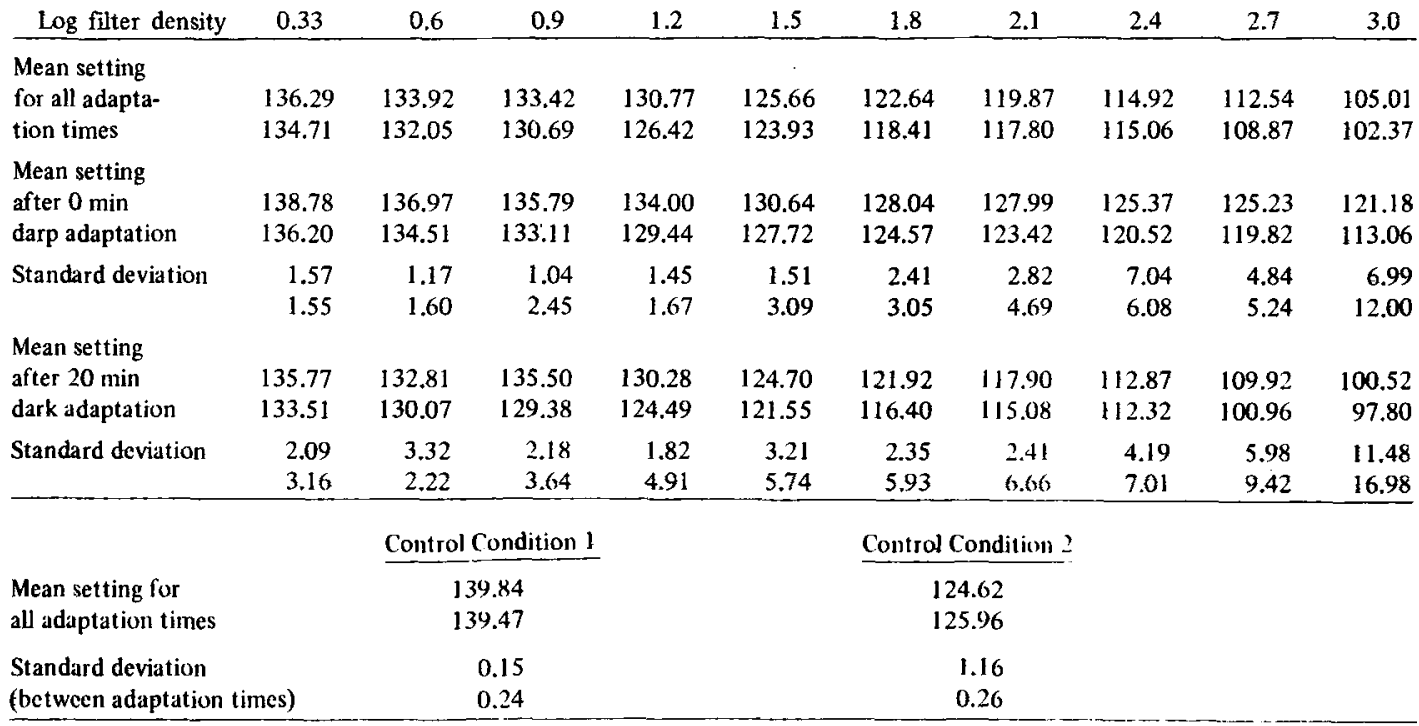


$p=0.05$ level if a one-tailed test is used, and the relationship must therefore be considered tenuous.

Does a similar adaptational mechanism underlie the deterioration in pattern vision observed both in this experiment and with retinal image stabilization? The conditions differ in two obvious respects: Firstly, there is no stabilization of images upon the retina when the $O$ inspects the inner surface of the eye cap. Secondly, while care is usually taken to ensure precise accommodation of the focus of the eye to the test figure in stabilized retinal image experiments, the eye caps used in this study are so close to the eye that the image is never sharply resolved, and the phenomenal appearance of a contour drawn upon the cap is of a blurred edge shading gradually into the background. This reduces the effect of small eye movements as the rate of change of luminance is less across a blurred contour than it is across a sharp one. There are also data from Enoch (1958) and Thomas and Kovar (1965) showing that perceived figure-ground contrast is reduced as the sharpness of the boundary separating figure from ground is decreased.

The results of this experiment can thus be accounted for in terms of lateral inhibitory interactions such as those observed in Limulus (Ratliff et al, 1963) and suggested for human vision (von Békésy, 1960). As the differentials in excitation between adjacent neural elements in the region of a diffuse contour would be small and relatively little change would occur as a result of micronystagmus the model would predict that after a time adaptation would be complete and all units would reach a uniform level of activity resulting in a loss of response to contour, as is the case in this experiment. As this adaptation takes time to occur it would also be expected that figure-ground differentials would be better maintained at low flicker frequencies. This prediction is sustained by the results of this experiment and also by Bittini et al (1960) who reported enhanced contrast of indefinitely contoured objects with intermittent illumination. An additional factor that would aid contour perception under flickering light is the reported increase in saccade rate induced at flicker frequencies in the region of 1 to 3 cps (West \& Boyce, 1968). Independent of the correctness of this explanation, the data clearly show that even large diffusely contoured figures on a ground of uniform brightness and texture can be perceptually unstable.

\section{REFERENCES}

BITTINI, M., ERCOLES, A. M., FIORENTINI, A., RONCHI, L., \& FRANCHIA, G. T. Enhanced contrast of an indefinitely contoured object by movement or intermittent illumination. Pubbl, Inst. naz. Office, 1960, $15,62-84$.

COHEN, W. Spatial and textural characteristics of the Ganzfeld. Amer. $J$. Psychol., 1957, 70, 403-410.

COHEN, W. Color perception in the chromatic Ganzfeld. Amer. J. Psychol, $1958,71,390-394$.

CORNSWEET, T. N. Determination of the stimuli for involuntary drifts and saccadic eye movements. J. Opt. Soc. Amer., 1956, 46, 987-993.

DITCHBURN, R. W., \& FENDER, D. H. The stabilized retinal image. Optica Acta, 1955, 2, 128-133.

DITCHBURN, R. W., \& PRITCHARD, R. M. Stabilized interference fringes on the retina. Nature, 1956, 177, 434.

ENOCH, J. M. Summated response of the retina to light entering different parts of the pupil. J. Opt. Soc. Amer., 1958, 48, 392-405.

EVANS, $C$. R. Some studies of pattern perception using a stabilized retinal image. Brit. J. Psychol., 1965, 56, 121-134.

FRY, G. A., \& ROBERTSON, V. M. The physiologic basis of the periodic merging of area into background. Amer. J. Psychol., 1935, 47, 644-655.

GUILFORD, J. P. Fluctuations of attention with weak visual stimuli. Amer. J. Psychol., 1927, 38, 534583.

KIRKWOOD, B. Relationship between the electroencephalogram, eye movements, and fluctuations of visual perception. Psychon. Sci., 1967, 7, 417-418.

RATLIFF, F., HARTLINE, H. K., \& MILLER, W. H. Spatial and temporal aspects of retinal inhibitory interaction. J. Opt. Soc. Amer., 1963, 53, 110-120.

RIGGS, L. A., RATLIFF, F., CORNSWEET, J. C., \& CORNSWEET, T. N. The disappearance of steadily fixated visual test objects. J. Opt. Soc. Amer., 1953, 43, 495-501.

THOMAS, J. P., \& KOVAR, C. W. The effect of contour sharpness on perceived brightness. Vision Res., 1965, 5, 559-564.

von BÉKÉSY, G. Neural inhibitory units of the eye and skin. Quantitative description of contrast phenomena. J. Opt. Soc. Amer., 1960, 50, 1060-1070.

WEST, D. C., \& BOYCE, P. R. Effect of flicker on eye movements. Vision Res., $1968,8,171-192$.

\section{NOTE}

1. Address: Department of Psychology, University of Otago, P. O. Box 56, Dunedin, N.Z.

(Accepted for publication on April 16, 1968.) 